\title{
Quality of life and related risk factors after breast reconstruction in breast cancer patients
}

\author{
Xiaoqing Wang ${ }^{1}$, Kepeng Zhu ${ }^{2}$, Liang Ren ${ }^{3}$, Hanbing Li ${ }^{1}$, Shuai Lin ${ }^{1}$, Xiao Qing ${ }^{1}$, Jinlian Wang ${ }^{4}$ \\ ${ }^{1}$ Department of Thyroid and Breast Surgery, Affiliated Hospital of North Sichuan Medical College, Nanchong 637000, China; ${ }^{2}$ Department of \\ General Surgery, Nanchong Central Hospital, Nanchong 637000, China; ${ }^{3}$ Department of Infection, Affiliated Hospital of North Sichuan Medical \\ College, Nanchong 637000, China; ${ }^{4}$ School of Nursing, North Sichuan Medical College, Nanchong 637000, China \\ Contributions: (I) Conception and design: X Wang, J Wang; (II) Administrative support: K Zhu, H Li; (III) Provision of study materials or patients: X \\ Wang, K Zhu, L Ren, S Lin; (IV) Collection and assembly of data: All authors; (V) Data analysis and interpretation: X Wang, L Ren, H Li, X Qing, J \\ Wang; (VI) Manuscript writing: All authors; (VII) Final approval of manuscript: All authors. \\ Correspondence to: Jinlian Wang. School of Nursing, North Sichuan Medical College, Nanchong 637000, China. Email: 2621083980@qq.com.
}

Background Breast cancer is the most frequently occurring cancer in women globally, using radical mastectomy as the main clinical treatment. This study aims to investigate the quality of life and related factors in patients after breast reconstruction.

Methods: Female patients undergoing breast reconstruction after radical mastectomy between February 20, 2014 and February 20, 2019 in the Department of Tumor or the Department of Thyroid and Breast Surgery in the Affiliated Hospital of North Sichuan Medical College and Nanchong Central Hospital were surveyed. The patients' basic information was collected using a questionnaire prepared by the research team, and the Functional Assessment of Cancer Therapy-Breast (FACT-B) questionnaire was used to assess the patients' quality of life. Multivariate linear regression analysis was performed to explore the factors impacting the change of FACT-B scores one year after surgery.

Results: A total of 150 patients were included. 143 of them had complete data, and 7 patients were lost to follow-up. Compared with the preoperative results, the scores of all dimensions of the FACT-B scale and the overall health status of the patients were significantly reduced at 3 months after surgery, which recovered to the preoperative level at 6 months after surgery $(\mathrm{P}<0.05)$, and significantly increased at 12 months after surgery $(\mathrm{P}<0.05)$. The results showed that age $\geq 40$ years, menopause, postoperative complications, and endocrine therapy were risk factors for decreased quality of life at 12 months after breast reconstruction. Meanwhile, tumor stage 0 or stage I and immediate breast reconstruction were protective factors for quality of life at 12 months after breast reconstruction $(\mathrm{P}<0.05)$.

Conclusions: The postoperative quality of life of patients with breast reconstruction is relatively high. Older age, post-menopause, postoperative complications, endocrine therapy, advanced tumor stage, and delayed reconstruction are all risk factors for decreased quality of life of patients. Targeted measures should be taken to improve the postoperative life quality of patients, such as paying more attention to the older or post-menopausal patients, as well as those with postoperative complications, endocrine therapy, high tumor stage, or delayed breast reconstruction.

Keywords: Breast cancer; breast reconstruction; quality of life

Submitted May 18, 2020. Accepted for publication Jun 15, 2020.

doi: $10.21037 /$ gs-20-532

View this article at: http://dx.doi.org/10.21037/gs-20-532 


\section{Introduction}

Breast cancer is the most common cancer among women worldwide, comprising more than $18 \%$ of all female malignancies (1). In China, the prevalence and incidence of breast cancer are increasing annually (2). The Global Burden of Disease Study 2019 reported that the prevalence of breast cancer among Chinese women in 2017 was 451.56 per 100,000, with an incidence rate of 51.72 per 100,000, bringing a considerable burden of disease to the country (2). At present, the clinical treatment of breast cancer mainly includes surgery, endocrine therapy, chemotherapy, radiotherapy and targeted therapy, but radical mastectomy is still the main clinical treatment for breast cancer patients; however, the mastectomy often results in axillary sag and chest wall damage after surgery, which can greatly impact the body image of the patient, leading to anxiety and depression, less social interaction, and consequently, significantly lower quality of life (3). Studies have shown that breast reconstruction can help to maintain the aesthetic appearance of the body, which can help to rebuild the patient's self-confidence, reduce their psychological trauma, and improve their quality of life $(4,5)$. However, a number of studies have reported that the life quality of women who undergo breast reconstruction after surgery is not higher than that of women who undergo pure breast resection (6). To date, research has only focused on evaluating the quality of life of patients before and after surgery, and there has been insufficient evaluation of the factors relating to longterm quality of life and changes in quality of life scores. Therefore, this study explored the factors influencing the changes in life quality after breast reconstruction in 150 breast cancer patients who were treated in the Affiliated Hospital of North Sichuan Medical College and Nanchong Central Hospital. We present the following article in accordance with the SURGE reporting checklist (available at http://dx.doi.org/10.21037/gs-20-532).

\section{Methods}

\section{General information}

A total of 150 female patients who underwent breast reconstruction (173 breasts) after radical mastectomy between February 20, 2014 and February 20, 2019 in the Department of Tumor or the Department of Thyroid and Breast Surgery in the Affiliated Hospital of North Sichuan Medical College and Nanchong Central Hospital were surveyed. The study was approved by the Ethics
Committee of the Affiliated Hospital of North Sichuan Medical College. The patients had an average age of $48.23 \pm 6.35$ (range, 22 to 61 ) years old. Among them, 69 patients received immediate breast reconstruction and 81 patients received delayed breast reconstruction. There were 12,52 , and 86 patients with tumors in stages 0 , I, and II, respectively. Postoperative pathology showed that there were 119 cases of invasive ductal carcinoma, 15 cases of mucinous adenocarcinoma, 8 cases of invasive lobular carcinoma, 6 cases of tubular carcinoma, and 2 cases of medullary carcinoma. All patients signed the informed consent form and agreed to follow-up before participating in the quality of life survey. The study was conducted in accordance with the Declaration of Helsinki.

\section{Inclusion and exclusion criteria}

The inclusion criteria for patients were as follows: (I) a diagnosis of breast cancer confirmed by pathological biopsy; (II) primary tumors of stages $0-\mathrm{II}$; (III) no anxiety or depression reported before the surgery, and no presence of other diseases that may affect quality of life; and (IV) a local resident.

The exclusion criteria were as follows: (I) severe complications in vital organs at the time of enrollment; (II) mental consciousness disease, language disorders, or dementia; (III) scar constitution; or (IV) other tumors present.

\section{Questionnaire survey}

The basic information of the patients was collected using a questionnaire prepared by the research team. The questionnaire recorded the name, age, education level, marital status, family income, menopausal status, and smoking and drinking history of the patients, as well as whether they had diabetes or hypertension. Relevant postoperative clinical characteristics of the patients were collected, including tumor stage, pathological type, tumor size, reconstruction type, and whether they had undergone postoperative radiotherapy, chemotherapy, or endocrine therapy.

The life quality of the patients was evaluated using the Functional Assessment of Cancer Therapy-Breast (FACT-B) questionnaire (7). The FACT-B questionnaire has high sensitivity for evaluating the physiological, emotional, and social function of breast cancer patients. The FACT-B evaluation system includes FACT-G, an evaluation of the 
Table 1 Comparison of the FACT-B scale scores of the patients before and after breast reconstruction surgery $(\bar{x} \pm s$, scores)

\begin{tabular}{lccccccc}
\hline Time & Cases & $\begin{array}{c}\text { Physiological } \\
\text { status }\end{array}$ & $\begin{array}{c}\text { Social/family } \\
\text { status }\end{array}$ & $\begin{array}{c}\text { Emotional } \\
\text { status }\end{array}$ & $\begin{array}{c}\text { Functional } \\
\text { status }\end{array}$ & $\begin{array}{c}\text { Additional } \\
\text { attention }\end{array}$ & $\begin{array}{c}\text { FACT-B scale total } \\
\text { scores }\end{array}$ \\
\hline Pre-operation & 150 & $22.57 \pm 2.04$ & $23.39 \pm 2.46$ & $17.19 \pm 3.25$ & $19.98 \pm 3.85$ & $25.63 \pm 2.91$ & $108.76 \pm 13.01$ \\
3 months postoperatively & 149 & $18.20 \pm 3.65^{*}$ & $19.36 \pm 3.07^{*}$ & $13.53 \pm 2.81^{*}$ & $13.79 \pm 2.56^{*}$ & $21.88 \pm 3.62^{*}$ & $86.76 \pm 11.28^{*}$ \\
6 months postoperatively & 149 & $22.35 \pm 4.16$ & $23.74 \pm 3.92$ & $17.81 \pm 3.39$ & $20.32 \pm 3.07$ & $26.22 \pm 3.56$ & $110.44 \pm 14.27$ \\
12 months postoperatively & 149 & $25.81 \pm 3.83^{*}$ & $25.68 \pm 2.91^{*}$ & $21.58 \pm 3.47^{*}$ & $21.25 \pm 4.14^{*}$ & $29.32 \pm 3.18^{\star}$ & $123.64 \pm 15.08^{*}$ \\
F & & 115.822 & 105.588 & 151.372 & 157.747 & 122.932 & 189.040 \\
P & & $<0.001$ & 0.001 & $<0.001$ & $<0.001$ & $<0.001$ & $<0.001$ \\
\hline
\end{tabular}

Note: compared with before surgery, ${ }^{*}, \mathrm{P}<0.05$. FACT-B, Functional Assessment of Cancer Therapy-Breast.

life quality of cancer patients and a subscale for breast cancer. The FACT-G scale comprises physiological status (7 items), social and family status (7 items), emotional status (6 items), and functional status (7 items), while the breast cancer subscale includes 9 items. The FACT-B scale has a total score of 144; the higher the score, the better the patient's quality of life (8). Cronbach's $\alpha$ coefficient of each dimension of the FACT-B scale in this survey was $0.63-0.84$, and the content validity index was 0.71 . In order to ensure the quality of the questionnaire, the medical staff in the survey have received unified training and assessment to ensure that each investigator correctly understands the criteria of each option in the questionnaire. The questionnaire can be filled out face to face during the survey and one-to-one through telephone during the followup. The questionnaire collected should be ensured the completeness and accuracy of the questionnaire on the spot, otherwise to correct or supplement the wrong or missing items found on the spot.

\section{Follow-up survey}

The patients were followed-up by outpatient visits or telephone at 3, 6, and 12 months after discharge. The questionnaire was completed in a question-and-answer format, and the patients were re-examined to determine whether there was recurrence, distant metastasis, abnormal breast prosthesis, or any other abnormalities.

\section{Statistical methods}

SPSS 22.0 statistical software (IBM, USA) was used for data analysis. The FACT-B scale scores of the breast reconstruction patients followed a normal distribution and were expressed as the mean \pm standard deviation $(\bar{x} \pm s)$. The FACT-B scale scores were compared at different times before and after surgery using linear mixed models to analyze non-balanced repeated measurement data. A univariate analysis of changes in the quality of life scores in the breast reconstruction patients at 12 months after surgery was performed using the independent-samples $t$-test, and the multivariate linear regression analysis was used for multivariate analysis. Results with $\mathrm{P}<0.05$ were considered statistically significant.

\section{Results}

\section{Comparison of FACT-B scale scores before and after surgery}

Of the 150 breast reconstruction patients surveyed, 7 were lost to follow-up due to loss of contact after a change of phone number. No relapses or deaths occurred among the remaining 143 patients. Compared with the preoperative results, all dimensions and overall health scores on the FACT-B scale were significantly reduced at 3 months postoperatively. However, at 6 months after surgery, they had returned to the preoperative level $(\mathrm{P}>0.05)$, and at 12 months after surgery, all scores were significantly increased (all $\mathrm{P}<0.05$ ). The detail information is shown in Table 1.

\section{Univariate analysis of differences in score before and after surgery}

At 12 months after surgery, the total score of the 143 patients on FACT-B scale was $(14.73 \pm 4.15)$ points higher than the total score before surgery. Univariate analysis 
showed that factors including age, family income, tumor stage, reconstruction type, menopausal status, having diabetes or not, experiencing postoperative complications or not, and receiving endocrine therapy or not were all factors that influenced differences in FACT-B scale score before and 12 months after breast reconstruction $(\mathrm{P}<0.05$, Table 2$)$.

\section{Analysis of multiple factors affecting the life quality of patients after breast reconstruction}

Multivariate linear regression analysis was performed. The difference in the total FACT-G scale scores was taken as the dependent variable, and the statistically significant factors in the univariate analysis were taken as the independent variables. The results revealed that factors such as age ( $\geq 40$ years old), menopause, postoperative complications, and endocrine therapy were risk factors affecting the quality of life of patients at 12 months after breast reconstruction. Meanwhile, having tumors at stages 0 or I and immediate breast reconstruction were protective factors for the quality of life of patients 12 months after breast reconstruction $(\mathrm{P}<0.05$, Table 3).

Table 2 Univariate analysis of the difference of the FACT-B scale scores of patients with breast reconstruction after surgery

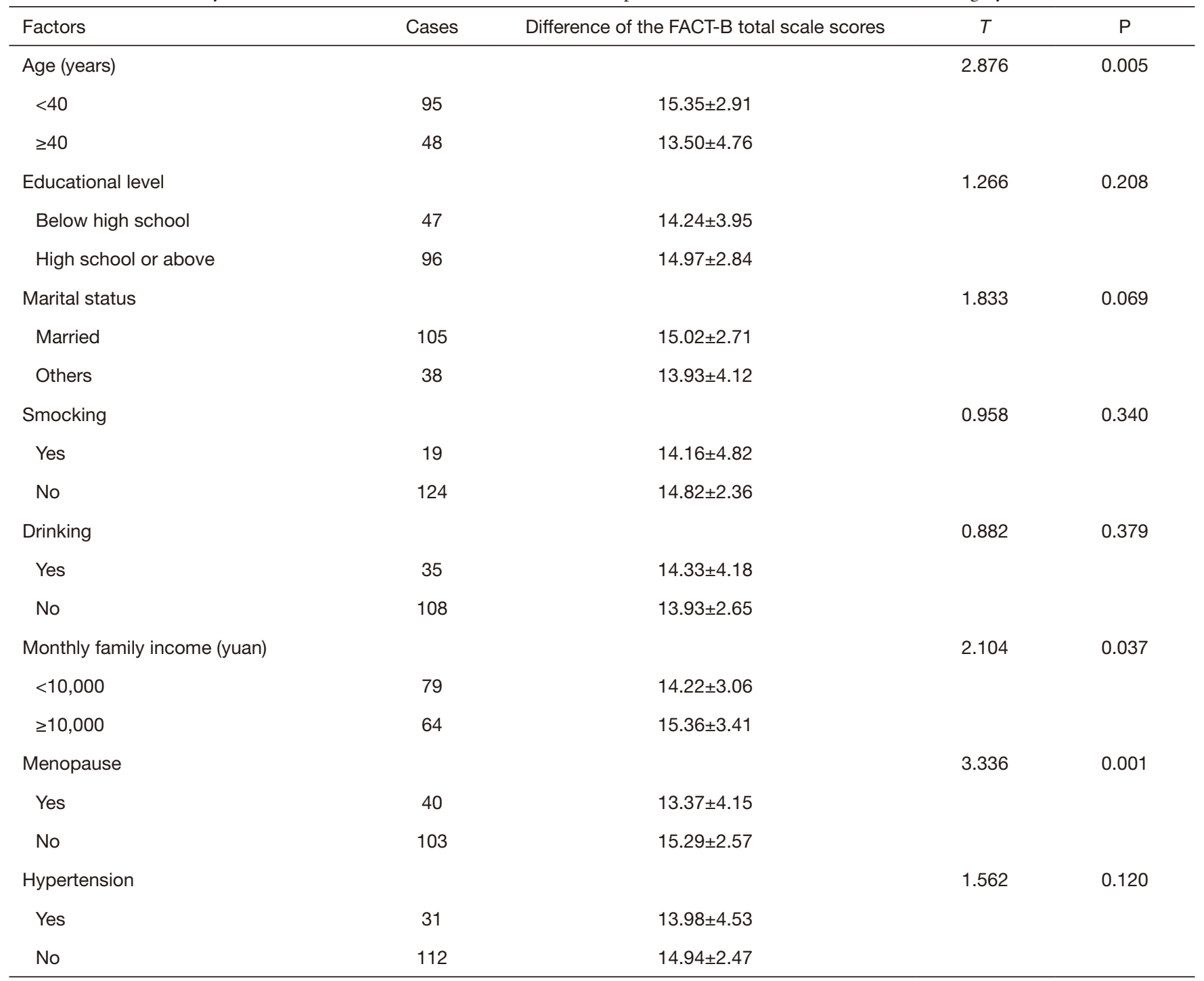

Table 2 (continued) 
Table 2 (continued)

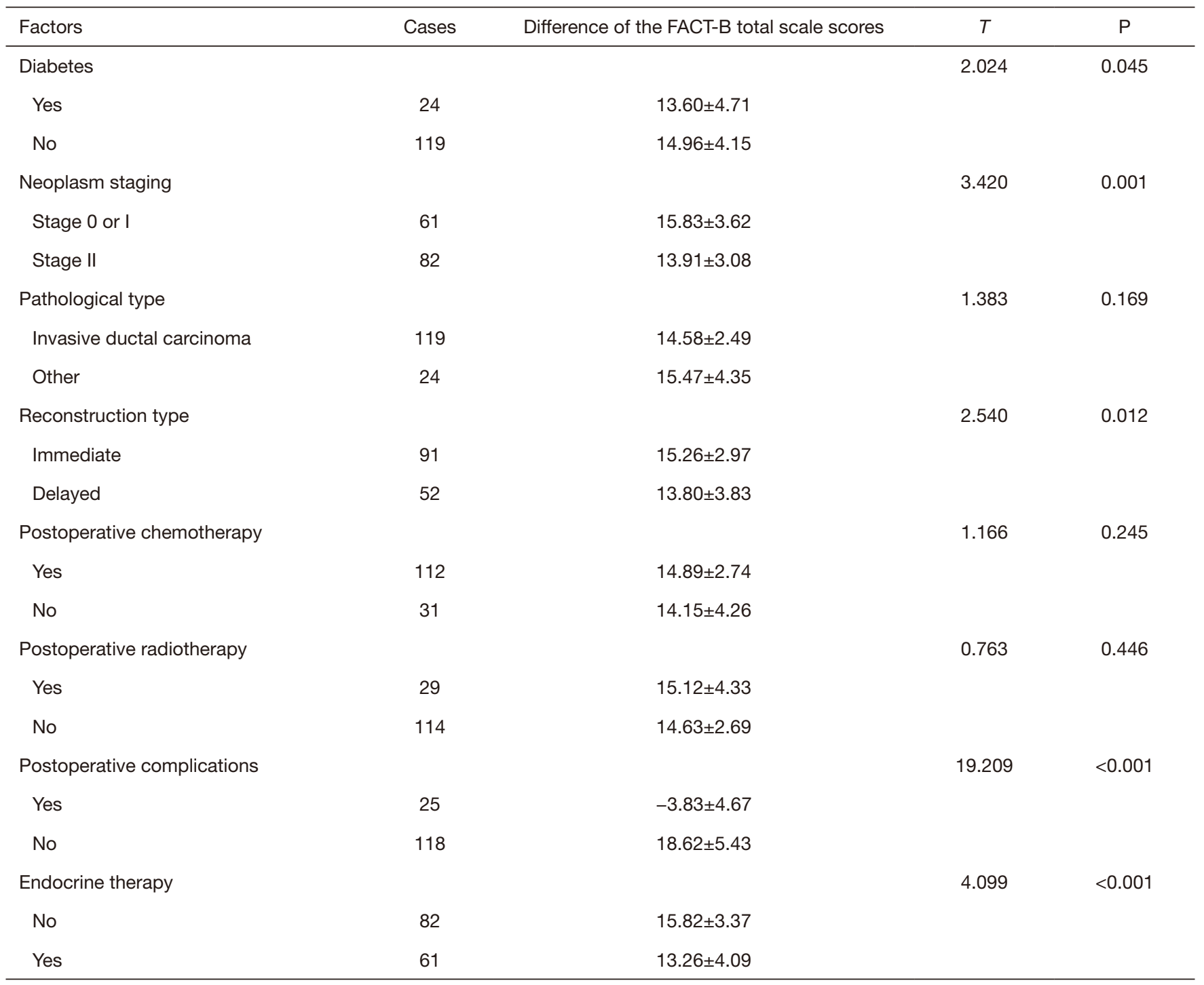

FACT-B, Functional Assessment of Cancer Therapy-Breast.

Table 3 Multivariate analysis of the differences in the FACT-B scale scores of patients with breast reconstruction after surgery

\begin{tabular}{lccccc}
\hline Factors & B & Standard error & $t$ & $\mathrm{P}$ & $95 \% \mathrm{Cl}$ \\
\hline Age (years) ( $\geq 40$ vs. $<40$ ) & -0.946 & 0.379 & -2.496 & 0.014 & $-1.689,-0.203$ \\
Monthly family income (yuan) $(\geq 10,000$ vs. $<10,000)$ & 0.721 & 0.345 & 2.090 & 0.038 & $0.045,1.397$ \\
Menopause (yes vs. no) & -0.322 & 0.116 & -2.776 & 0.006 & $-0.549,-0.095$ \\
Neoplasm staging (stage 0 or I vs. stage II) & 1.127 & 0.358 & 3.148 & 0.002 & $0.425,1.829$ \\
Reconstruction type (immediate vs. delayed) & 0.805 & 0.294 & 2.738 & 0.007 & $0.229,1.381$ \\
Postoperative complications (yes vs. no) & -1.245 & 0.351 & -3.547 & 0.001 & $-1.933,-0.557$ \\
Endocrine therapy (yes vs. no) & -0.407 & 0.192 & -2.120 & 0.036 & $-0.783,-0.031$ \\
\hline
\end{tabular}

FACT-B, Functional Assessment of Cancer Therapy-Breast. 


\section{Discussion}

Breast cancer has become the most frequently occurring cancer in young women between the ages of 15 and 39. Due to the strict requirements for breast-conserving treatment, radical mastectomy is the main treatment for breast cancer patients. With the transformation of the bio-psychosocial medical model and the improvement of individuals' living standards, the life quality of patients after surgery has received an increasing amount of attention. Studies have shown that the quality of life is significantly higher in patients of breast-conserving and breast reconstruction surgery than in patients who undergo pure breast resection. Moreover, the life quality of patients who receive breast reconstruction is higher than that of patients who do not undergo breast reconstruction, and there is no significant difference in the overall survival rates of the two groups (9-11). In Western countries, breast reconstruction surgery now constitutes an important surgical part of breast cancer treatment (12).

The FACT-B questionnaire has been confirmed as a reliable and valid method for investigating the life quality of breast cancer patients in China. This study suggested that the patients' scores for physical status, social/family status, emotional status, functional status, and overall health status at 3 months postoperatively were significantly lower than those before surgery. This decrease can be attributed to the side effects of radiotherapy and chemotherapy, lower physical energy, and bodily comfort during postoperative recovery and adaptation, as well as the worry patients often feel about the efficacy of their treatment and the possibility of recurrence or metastases after surgery. At 6 months after surgery, the quality of life scores of the patients had returned to their preoperative level, which indicates that after 6 months of adaptation and rehabilitation, the patients' quality of life in terms of physiological status, family, and emotions had started to recover. At 12 months after surgery, the FACT- B scale scores were increased and were significantly higher than the scores before surgery. It has been reported that anxiety, depression and negative emotions of patients at 12 and 18 months after surgery are significantly reduced, and the quality of life is improved, which is similar to the results of this study (13).

In this study, 150 patients were followed up for 1 year without recurrence or metastasis, and 143 patients who completed the 12-month follow-up. Age $\geq 40$ years, menopause, postoperative complications, and endocrine therapy were found to be risk factors affecting the quality of life of patients 12 months after breast reconstruction. Studies have found that women aged over 40 often have low libido, which may be one of the reasons for the decline in their quality of life (14). Meanwhile, elderly patients often worry about their survival time, the risk of future generations developing the same disease, and other psychological burdens, resulting in a decrease in their quality of life. Menopausal women often suffer symptoms such as mood swings and postural changes caused by endocrine disorders, as well as sexual dysfunction, all of which affect life quality. Furthermore, menopausal women are often older, which introduces another risk factor for menopausal patients who undergo breast reconstruction. Therefore, more attention should be paid to older women after surgery. In the process of clinical nursing and follow-up, we should communicate more with elderly female patients to relieve their worries and anxiety in time, which is conducive to improving the quality of life of patients. The results of this study also found that endocrine therapy was associated with a decrease in the quality of life of patients. This supports the findings of another study, in which a high proportion of patients with endocrine therapy had sexual dysfunction, resulting in a decrease in quality of life (15), and is also similar to the conclusion of Wang et al. in China (16). The main complications of breast reconstruction surgery are postoperative flap infection, incision infection, prosthesis ectopic, and external leakage, which cause pain and significantly decrease the patients' quality of life. This study showed that at 12 months after breast reconstruction, tumors at stage 0 or I and immediate breast reconstruction were the protective factors for the life quality of patients. Tumor stage affects the prognosis of the patient, as well as their treatment plan and psychological outlook. The lower the tumor stage, the lower the impact on the patient's life and the higher the quality of life score. Studies have shown that patients with delayed breast reconstruction feel more stress in relation to body image, emotions, physiology, and psychology, and a higher proportion of these patients experience anxiety and depression compared with patients who receive breast reconstruction immediately $(13,17)$. Clinically, It is suggested to conduct more personalized follow-up guidance, pay attention to humanistic care, timely discover patients psychological contradictions and negative emotions, keep close communication with the family members at the same time, in order to obtain the actively support of family members, improve the patient' confidence of their body and the level sexual regulation, consciously guiding the formation of the family relationship, promoting 
the quality of life of postoperative patients.

In this study, factors related to the quality of life of patients were collected through questionnaire. Although the FACT-B questionnaire has a high reliability and validity, subjective factors of patients also have an impact on the questionnaire results. At the same time, due to the short follow-up time of patients, the long-term impact, such as 5 years later, cannot be evaluated, so the test results need to be verified by long-term follow-up results.

In summary, the quality of life of patients who undergo breast reconstruction is well recovered at 6 months after surgery. Age, menopause, postoperative complications, endocrine therapy, tumor stage, and reconstruction time are the main factors affecting the quality of life scores of breast cancer patients at 12 months after breast reconstruction. Therefore, clinically, more attention should be paid to the above groups and take personalized follow-up guidance, which is conducive to improving patients' postoperative quality of life.

\section{Acknowledgments}

Funding: None.

\section{Footnote}

Reporting Checklist: The authors have completed the SURGE reporting checklist. Available at http://dx.doi. org/10.21037/gs-20-532

Data Sharing Statement: Available at http://dx.doi. org/10.21037/gs-20-532

Conflicts of Interest: All authors have completed the ICMJE uniform disclosure form (available at http://dx.doi. org/10.21037/gs-20-532). The authors have no conflicts of interest to declare.

Ethical Statement: The authors are accountable for all aspects of the work in ensuring that questions related to the accuracy or integrity of any part of the work are appropriately investigated and resolved. The study was approved by the Ethics Committee of the Affiliated Hospital of North Sichuan Medical College (No. 2020ER(N)060-1). All patients signed the informed consent form and agreed to follow-up before participating in the quality of life survey. The study was conducted in accordance with the Declaration of Helsinki.
Open Access Statement: This is an Open Access article distributed in accordance with the Creative Commons Attribution-NonCommercial-NoDerivs 4.0 International License (CC BY-NC-ND 4.0), which permits the noncommercial replication and distribution of the article with the strict proviso that no changes or edits are made and the original work is properly cited (including links to both the formal publication through the relevant DOI and the license). See: https://creativecommons.org/licenses/by-nc-nd/4.0/.

\section{References}

1. Naito Y, Urasaki T. Precision medicine in breast cancer. Chin Clin Oncol 2018;7:29.

2. Global Burden of Disease Study 2019. Results. Seattle, United States: Institute for Health Metrics and Evaluation (IHME). Available online: http://ghdx.healthdata.org/gbdresults-tool (2019).

3. Salibian AA, Frey JD, Karp NS. Strategies and considerations in selecting between subpectoral and prepectoral breast reconstruction. Gland Surg 2019;8:11-8.

4. Eltahir Y, Krabbe-Timmerman IS, Sadok N, et al. Outcome of Quality of Life for Women Undergoing Autologous versus Alloplastic Breast Reconstruction following Mastectomy: A Systematic Review and MetaAnalysis. Plast Reconstr Surg 2020;145:1109-23.

5. Guo R, Wu J. Current status and progress of oncoplastic surgery and breast reconstruction after breast cancer. Chin J Surg Onco 2018;10:141-6.

6. Siqueira HFF, Teixeira JLA, Lessa Filho RDS, et al. Patient satisfaction and quality of life in breast reconstruction: assessment of outcomes of immediate, delayed, and nonreconstruction. BMC Res Notes 2020;13:223.

7. Cella DF, Tulsky DS, Gray G, et al. The functional assessment of cancer therapy scale: development and validation of the general measure. J Clin Oncol 1993;11:570-9.

8. Wan CH, Zhang DM, Tang XL, et al. Revision of the Chinese version of the FACT-B for patients with breast cancer. Chinese Mental Health Journal 2003;17:298-300.

9. Metcalfe KA, Semple J, Quan ML, et al. Changes in psychosocial functioning 1 year after mastectomy alone, delayed breast reconstruction, or immediate breast reconstruction. Ann Surg Oncol 2012;19:233-41.

10. Dauplat J, Kwiatkowski F, Rouanet P, et al. Quality of life after mastectomy with or without immediate breast reconstruction. Br J Surg 2017;104:1197-206.

11. Baker JL, Mailey B, Tokin CA, et al. Postmastectomy 
reconstruction is associated with improved survival in patients with invasive breast cancer: a single-institution study. Am Surg 2013;79:977-81.

12. Driul L, Bernardi S, Bertozzi S, et al. New surgical trends in breast cancer treatment: conservative interventions and oncoplastic breast surgery. Minerva Ginecol 2013;65:289-96.

13. Zhong T, Hu J, Bagher S, et al. A Comparison of Psychological Response, Body Image, Sexuality, and Quality of Life between Immediate and Delayed Autologous Tissue Breast Reconstruction: A Prospective Long-Term Outcome Study. Plast Reconstr Surg 2016;138:772-80.

14. Morrow PK, Broxson AC, Munsell MF, et al. Effect of age and race on quality of life in young breast cancer survivors.
Clin Breast Cancer 2014;14:e21-e31.

15. Safarinejad MR, Shafiei N, Safarinejad S. Quality of life and sexual functioning in young women with early-stage breast cancer 1 year after lumpectomy. Psychooncology 2013;22:1242-8.

16. Wang XJ, Zhang HL, Sun Q, et al. Analysis of life quality and correlating factors in patients with post radi- cal mastectomy and delayed-immediate breast reconstruction. China Medical Herald 2016;13:47-51.

17. Rosson GD, Shridharani SM, Magarakis M, et al. Quality of life before reconstructive breast surgery: A preoperative comparison of patients with immediate, delayed, and major revision reconstruction. Microsurgery 2013;33:253-8.

(English Language Editor: J. Reynolds)
Cite this article as: Wang $\mathrm{X}$, Zhu K, Ren L, Li H, Lin S, Qing X, Wang J. Quality of life and related risk factors after breast reconstruction in breast cancer patients. Gland Surg 2020;9(3):767-774. doi: 10.21037/gs-20-532 